\title{
Second-harmonic generation in an infinite layered structure with nonlinear spring-type interfaces
}

\section{$\operatorname{AUTHOR}(\mathrm{S})$ :}

Biwa, Shiro; Ishii, Yosuke

\section{CITATION:}

Biwa, Shiro ...[et al]. Second-harmonic generation in an infinite layered structure with nonlinear spring-type interfaces. Wave Motion 2016, 63: 55-67

\section{ISSUE DATE:}

2016-06-01

URL:

http://hdl.handle.net/2433/226600

\section{RIGHT:}

(c) 2016. This manuscript version is made available under the CC-BY-NC-ND 4.0 license

http://creativecommons.org/licenses/by-nc-nd/4.0/; The full-text file will be made open to the public on 01 June 2018 in accordance with publisher's 'Terms and Conditions for Self-Archiving'.; この論文は出版社版でありません。引用の際 には出版社版をご確認ご利用ください。; This is not the published version. Please cite only the published version. 


\title{
Second-harmonic generation in an infinite layered structure with nonlinear spring-type interfaces
}

\author{
Shiro Biwa* and Yosuke Ishii \\ Department of Aeronautics and Astronautics, Graduate School of Engineering, Kyoto University \\ Katsura, Nishikyo-ku, Kyoto 615-8540, Japan
}

\begin{abstract}
The second-harmonic generation characteristics in the elastic wave propagation across an infinite layered structure consisting of identical linear elastic layers and nonlinear spring-type interlayer interfaces are analyzed theoretically. The interlayer interfaces are assumed to have identical linear interfacial stiffness but can have different quadratic nonlinearity parameters. Using a perturbation approach and the transfer-matrix method, an explicit analytical expression is derived for the second-harmonic amplitude when the layered structure is impinged by a monochromatic fundamental wave. The analysis shows that the second-harmonic generation behavior depends significantly on the fundamental frequency reflecting the band structure of the layered structure. Two special cases are discussed in order to demonstrate such dependence, i.e., the second-harmonic generation by a single nonlinear interface as well as by multiple consecutive nonlinear interfaces. In particular, when the second-harmonic generation occurs at multiple consecutive nonlinear interfaces, the cumulative growth of the second-harmonic amplitude with distance is only expected in certain frequency ranges where the fundamental as well as the double frequencies belong to the pass bands of the layered structure. Furthermore, a remarkable increase of the second-harmonic amplitude is found near the terminating edge of pass bands. Approximate expressions for the low-frequency range are also obtained, which show the cumulative growth of the second-harmonic amplitude with quadratic frequency dependence.
\end{abstract}

Key Words: Layered structure; Nonlinear acoustics; Harmonic generation; Perturbation analysis; Spring-type interface; Band structure

* Corresponding author: Shiro Biwa, Department of Aeronautics and Astronautics, Graduate School of Engineering, Kyoto University, Katsura, Nishikyo-ku, Kyoto 615-8540, Japan, Tel: +81 75383 3796, E-mail: biwa@kuaero.kyoto-u.ac.jp 


\section{Introduction}

Contacting as well as weakly bonded interfaces between solid bodies are often quite different from perfectly bonded interfaces in their behavior when they interact with elastic waves. Such imperfect interfaces are present in various forms in nature as well as in technological products. Acoustic or ultrasonic characterization of imperfect interfaces, e.g., fractures in rocks [1], machine elements with contacting parts [2, 3], kissing bonds and closed cracks in structural members [4-6], has been studied extensively in geophysical exploration and ultrasonic nondestructive testing. When elastic waves impinge on these imperfect interfaces, they exhibit partial reflection and transmission in a frequency-dependent manner. Foregoing studies have revealed that such characteristics can be described by modeling the interfacial region as a spring-type interface characterized by normal and tangential stiffnesses [7-11]. Spring-type interface models have been adopted to analyze the response of closed cracks for different dynamic excitation conditions $[12,13]$. Waveguide characteristics of imperfect interfaces have been analyzed based on such models and used to characterize them [14-17]. Elastic wave propagation in multilayered structures with spring-type interlayer interfaces has also been analyzed in order to clarify the effect of thin interlaminar resin-rich regions in fiber-reinforced composite laminates [18-23].

Another prominent feature of imperfect interfaces is acoustic nonlinearity [24, 25]. In particular, contacting interfaces have been shown to produce significant higher-harmonic components when insonified by narrow-band, high-amplitude waves [26-28]. These features have attracted much attention in the field of ultrasonic nondestructive testing as a sensitive measure to detect closed defects and weak bonding in structural elements. Nonlinear spring-type interface models have been employed to predict the second-harmonic amplitude generated at a contacting interface between solids in reasonable agreement with experimental results [29-34]. In order to examine the second-harmonic generation behavior at a kissing bond interface in an adhesive joint, Yan et al. [35, 36] analyzed the one-dimensional wave propagation across an adhesive layer bonded to the adherend using the nonlinear spring-type interface model. They found that the second-harmonic amplitude to be observed is highly dependent on the ratio of the wavelength to the layer thickness due to the band-pass filtering effect of the layer. Ishii and Biwa [37] numerically showed that the second-harmonic generation behavior in multilayered structures with nonlinear spring-type interfaces exhibits remarkably complex frequency dependence. These findings indicate the importance of further examining the second-harmonic generation in multilayered structures due to interfacial nonlinear effects.

Recently, nonlinear elastic wave propagation in multilayered structures has been studied theoretically by different authors. Most of the foregoing studies for this topic consider periodic layered structures consisting of nonlinear elastic materials to explore the amplitude-dependent dispersion and band-gap characteristics [38,39] or the possible occurrence of localized solutions [40-42]. The 
second-harmonic generation in multilayered structures due to material nonlinearity was analyzed by Yun et al. [43]. It appears, however, that the corresponding issue due to interfacial nonlinearity has rarely been addressed in the existing literature. Better understanding of this issue is not only of academic interest but also expected to give insight into the characterization of weakly bonded structures, e.g., ultrasonic testing of composite laminates with closed delaminations [44, 45], acoustic characterization of granular media [46, 47], etc.

The aim of this paper is to analyze the second-harmonic generation in the one-dimensional wave propagation across a multilayered structure with nonlinear spring-type interfaces. For a single nonlinear spring-type interface between semi-infinite elastic media, a perturbation analysis for the second-harmonic generation was presented by Biwa et al. [30]. In the present analysis, this foregoing analysis is extended to the case of multiple interfaces in layered structures. To gain fundamental understanding, our attention is focused on an infinite layered structure made of identical linear elastic layers and weakly nonlinear spring-type interfaces with identical linear interfacial stiffness. In Section 2 , the formulation is laid down and a perturbation approach is employed to linearize the governing equations. The solution to the fundamental wave propagation is outlined in Section 3 in the frequency domain using the transfer-matrix method [48, 49]. The corresponding problem for the second-harmonic component generated by nonlinear interfaces is analyzed in Section 4, and a formal analytical expression for the second-harmonic amplitude in the layered structure is obtained. In Section 5, two special cases are discussed. Namely, the second-harmonic generation characteristics by a single nonlinear interface as well as by multiple consecutive nonlinear interfaces are examined in detail.

\section{Formulation}

The one-dimensional longitudinal wave propagation across an infinitely extended multilayered structure made of identical linear elastic layers (mass density $\rho$, wave velocity $c$ and thickness $h$ ), which are bonded to each other by spring-type interfaces, is considered as shown in Fig. 1. The wave is assumed to propagate in the direction perpendicular to the layers. The displacement $u(x, t)$ is a function of the position $x$ and time $t$, and obeys the linear equation of motion in each layer, i.e.,

$$
\frac{\partial^{2} u}{\partial t^{2}}=c^{2} \frac{\partial^{2} u}{\partial x^{2}}, X_{m}<x<X_{m+1},
$$

where $X_{m}=m h(m=0, \pm 1, \pm 2, \ldots \ldots)$ denote the positions of interlayer interfaces. At each interface, the stress $\sigma(x, t)=\rho c^{2} \partial u / \partial x$ is continuous while discontinuity is allowed in the displacement. When the spring-type interfaces possess weak quadratic nonlinearity [30], the boundary conditions at $x=X_{m}$ are given by 


$$
\rho c^{2} \frac{\partial u}{\partial x}\left(X_{m+}, t\right)=\rho c^{2} \frac{\partial u}{\partial x}\left(X_{m-}, t\right)=K_{\mathrm{S}}\left[1-\beta_{m} y_{m}(t)\right] y_{m}(t),
$$

where $K_{\mathrm{S}}$ is the linear interfacial stiffness, assumed to be the same for all interfaces, and $\beta_{m}$ is a positive parameter representing the interfacial nonlinearity of the interface at $x=X_{m}$. Furthermore,

$$
y_{m}(t)=u\left(X_{m+}, t\right)-u\left(X_{m-}, t\right)
$$

denotes the jump of displacement at $x=X_{m}$. The subscripts " $m+$ " and " $m-$ " denote the limit of a field variable when $x$ approaches $X_{m}$ from the right and the left, respectively.

By introducing a characteristic frequency $\omega_{0}$ and a displacement amplitude $a_{0}$, the governing equations are written in terms of non-dimensional variables as

$$
\begin{aligned}
& \frac{\partial^{2} \tilde{u}}{\partial \tilde{t}^{2}}=\frac{\partial^{2} \tilde{u}}{\partial \tilde{x}^{2}}, \quad \tilde{X}_{m}<\tilde{x}<\tilde{X}_{m+1}, \\
& \frac{\partial \widetilde{u}}{\partial \widetilde{x}}\left(\tilde{X}_{m+}, \tilde{t}\right)=\frac{\partial \widetilde{u}}{\partial \widetilde{x}}\left(\widetilde{X}_{m-}, \widetilde{t}\right)=\widetilde{K}\left[1-\varepsilon_{m} \tilde{y}_{m}(\widetilde{t})\right] \tilde{y}_{m}(\widetilde{t}), \\
& \tilde{y}_{m}(\tilde{t})=\tilde{u}\left(\tilde{X}_{m+}, \tilde{t}\right)-\tilde{u}\left(\tilde{X}_{m-}, \widetilde{t}\right),
\end{aligned}
$$

where

$$
\tilde{x}=\frac{\omega_{0}}{c} x, \tilde{X}_{m}=\frac{\omega_{0}}{c} X_{m}, \tilde{t}=\omega_{0} t, \tilde{u}(\tilde{x}, \tilde{t})=\frac{u(x, t)}{a_{0}}, \tilde{K}=\frac{K_{\mathrm{S}}}{\rho c \omega_{0}}, \varepsilon_{m}=\beta_{m} a_{0} .
$$

Assuming that $\varepsilon=\operatorname{Max}_{m}\left[\varepsilon_{m}\right]$ is a sufficiently small quantity, the solution $\widetilde{u}(\widetilde{x}, \tilde{t})$ is expanded into a power series with respect to $\varepsilon$ as

$$
\tilde{u}(\tilde{x}, \tilde{t})=\tilde{u}_{1}(\tilde{x}, \tilde{t})+\varepsilon \tilde{u}_{2}(\tilde{x}, \tilde{t})+\varepsilon^{2} \tilde{u}_{3}(\tilde{x}, \tilde{t})+\cdots \cdots,
$$

and substituted into Eqs. (4)-(6). Collecting the terms for each order of $\varepsilon$ gives,

$$
\begin{aligned}
& \frac{\partial^{2} \tilde{u}_{j}}{\partial \tilde{t}^{2}}=\frac{\partial^{2} \tilde{u}_{j}}{\partial \tilde{x}^{2}}, \tilde{X}_{m}<\tilde{x}<\tilde{X}_{m+1}, \\
& \tilde{y}_{j m}(\tilde{t})=\tilde{u}_{j}\left(\tilde{X}_{m+}, \tilde{t}\right)-\tilde{u}_{j}\left(\tilde{X}_{m-}, \tilde{t}\right),
\end{aligned}
$$

for $j=1,2, \cdots$, and

$$
\begin{aligned}
& \text { For } \varepsilon^{0} ; \frac{\partial \widetilde{u}_{1}}{\partial \widetilde{x}}\left(\tilde{X}_{m+}, \tilde{t}\right)=\frac{\partial \widetilde{u}_{1}}{\partial \widetilde{x}}\left(\tilde{X}_{m-}, \tilde{t}\right)=\widetilde{K} \tilde{y}_{1 m}(\tilde{t}), \\
& \text { For } \varepsilon^{1} ; \frac{\partial \tilde{u}_{2}}{\partial \widetilde{x}}\left(\widetilde{X}_{m+}, \tilde{t}\right)=\frac{\partial \widetilde{u}_{2}}{\partial \widetilde{x}}\left(\tilde{X}_{m-}, \tilde{t}\right)=\widetilde{K}\left[\tilde{y}_{2 m}(\widetilde{t})-\delta_{m} \widetilde{y}_{1 m}(\widetilde{t})^{2}\right] \text {, } \\
& \text { For } \varepsilon^{2} ; \frac{\partial \tilde{u}_{3}}{\partial \widetilde{x}}\left(\widetilde{X}_{m+}, \widetilde{t}\right)=\frac{\partial \tilde{u}_{3}}{\partial \widetilde{x}}\left(\widetilde{X}_{m-}, \widetilde{t}\right)=\widetilde{K}\left[\widetilde{y}_{3 m}(\widetilde{t})-2 \delta_{m} \widetilde{y}_{1 m}(\tilde{t}) \tilde{y}_{2 m}(\tilde{t})\right] \text {, }
\end{aligned}
$$

and so on, where $\delta_{m}=\varepsilon_{m} / \varepsilon$. The problem given by Eqs. (4)-(6) can then be solved in a successive 
manner starting with the linear problem of Eqs. (9) and (10) with the boundary conditions of Eq. (11a), and then those of the next higher order in $\varepsilon$, which are also linear but contain an inhomogeneous term consisting of the solutions to the problems of the lower orders. In the present analysis, solutions are sought for to the problems for $\varepsilon^{0}$ and $\varepsilon^{1}$ in order to examine the second-harmonic generation behavior when the multilayered structure is subjected to a monochromatic wave propagation of angular frequency $\omega_{0}$.

\section{Propagation of monochromatic fundamental wave}

\subsection{Transfer-matrix formulation}

The problem of Eqs. (9)-(11a) is rewritten in terms of dimensional variables as

$$
\begin{aligned}
& \frac{\partial^{2} u_{1}}{\partial t^{2}}=c^{2} \frac{\partial^{2} u_{1}}{\partial x^{2}}, X_{m}<x<X_{m+1}, \\
& \rho c^{2} \frac{\partial u_{1}}{\partial x}\left(X_{m+}, t\right)=\rho c^{2} \frac{\partial u_{1}}{\partial x}\left(X_{m-}, t\right)=K_{\mathrm{S}} y_{1 m}(t),
\end{aligned}
$$

where $u_{1}(x, t)=a_{0} \tilde{u}_{1}(\tilde{x}, \tilde{t})$ and $y_{1 m}(t)=u_{1}\left(X_{m+}, t\right)-u_{1}\left(X_{m-}, t\right)$. Now that the nonlinear terms are not involved in Eq. (13), the layered structure considered in this problem possesses perfect periodicity of spatial period $h$. A solution to this problem, corresponding to the monochromatic wave propagation with angular frequency $\omega_{0}$ in the periodic layered structure, is described below. By introducing the complex-value expressions

$$
\begin{aligned}
& u_{1}(x, t)=\operatorname{Re}\left[U_{1}(x) \exp \left(-\mathrm{i} \omega_{0} t\right)\right], \\
& y_{1 m}(t)=\operatorname{Re}\left[Y_{1 m} \exp \left(-\mathrm{i} \omega_{0} t\right)\right],
\end{aligned}
$$

the problem is formulated in the frequency domain as

$$
\begin{aligned}
& \frac{d^{2} U_{1}}{d x^{2}}+\frac{\omega_{0}^{2}}{c^{2}} U_{1}=0, X_{m}<x<X_{m+1}, \\
& \rho c^{2} \frac{d U_{1}}{d x}\left(X_{m+}\right)=\rho c^{2} \frac{d U_{1}}{d x}\left(X_{m-}\right)=K_{\mathrm{S}} Y_{1 m} .
\end{aligned}
$$

The displacement solution to Eq. (15) is given as the sum of the forward-propagating wave $U_{1 \mathrm{~F}}(x)$ and the backward-propagating wave $U_{1 \mathrm{~B}}(x)$ in each layer, i.e., $U_{1}(x)=U_{1 \mathrm{~F}}(x)+U_{1 \mathrm{~B}}(x)$. Below, these wave components are denoted as the vector $\mathbf{U}_{1}(x)=\left(U_{1 \mathrm{~F}}(x), U_{1 \mathrm{~B}}(x)\right)^{\mathrm{T}}$. Note that $U_{1}(x)$ and $\mathbf{U}_{1}(x)$ are not to be confused, as the former is the displacement and the latter is a vector consisting of its forward- and backward-propagating components. Using the classical transfer-matrix formalism [48, 49], the components at both sides of the interface at $x=X_{m+1}$ are related by

$$
\mathbf{U}_{1}\left(X_{(m+1)+}\right)=\mathbf{S}(\Omega) \mathbf{U}_{1}\left(X_{(m+1)-}\right),
$$


where $\Omega=\omega_{0} h / c$ is the normalized angular frequency and the matrix $\mathbf{S}(\Omega)$ is defined by $[18,19$, 21]

$$
\mathrm{S}(\Omega)=\left[\begin{array}{cc}
1+\mathrm{i} \Lambda \Omega & -\mathrm{i} \Lambda \Omega \\
\mathrm{i} \Lambda \Omega & 1-\mathrm{i} \Lambda \Omega
\end{array}\right], \quad \Lambda=\frac{\rho c^{2}}{2 K_{\mathrm{S}} h} .
$$

The non-dimensional parameter $\Lambda$ defined above characterizes the relative linear compliance of the interface. Furthermore, $\mathbf{U}_{1}\left(X_{(m+1)_{-}}\right)$is connected to $\mathbf{U}_{1}\left(X_{m+}\right)$ by the propagator matrix $\mathbf{P}(\Omega)$ as

$$
\mathbf{U}_{1}\left(X_{(m+1)-}\right)=\mathbf{P}(\Omega) \mathbf{U}_{1}\left(X_{m+}\right),
$$

where

$$
\mathbf{P}(\Omega)=\left[\begin{array}{cc}
\exp (\mathrm{i} \Omega) & 0 \\
0 & \exp (-\mathrm{i} \Omega)
\end{array}\right] .
$$

Therefore, the wave components across one unit cell of periodic structure are related by

$$
\mathbf{U}_{1}\left(X_{(m+1)+}\right)=\mathbf{H}(\Omega) \mathbf{U}_{1}\left(X_{m+}\right),
$$

where

$$
\mathbf{H}(\Omega) \equiv \mathbf{S}(\Omega) \mathbf{P}(\Omega)=\left[\begin{array}{cc}
(1+\mathrm{i} \Lambda \Omega) \exp (\mathrm{i} \Omega) & -\mathrm{i} \Lambda \Omega \exp (-\mathrm{i} \Omega) \\
\mathrm{i} \Lambda \Omega \exp (\mathrm{i} \Omega) & (1-\mathrm{i} \Lambda \Omega) \exp (-\mathrm{i} \Omega)
\end{array}\right]
$$

is the transfer matrix for the unit cell.

\subsection{Application of Bloch theorem}

For the periodic structure considered here, the so-called Bloch theorem implies

$$
\mathbf{U}_{1}\left(X_{(m+1)+}\right)=\exp [\mathrm{i} K(\Omega) h] \mathbf{U}_{1}\left(X_{m+}\right),
$$

where $K(\Omega)$ denotes the Bloch wave number in the periodic structure at angular frequency $\omega_{0}$. Combined with Eq. (21), it is clear that $\exp [\mathrm{i} K(\Omega) h]$ is an eigenvalue of $\mathbf{H}(\Omega)$. After some manipulation and introducing an auxiliary function $G(\Omega)$ as

$$
G(\Omega) \equiv \cos \Omega-\Lambda \Omega \sin \Omega,
$$

two eigenvalues of $\mathbf{H}(\Omega)$ can be expressed as

$$
\begin{aligned}
& \exp \left[\mathrm{i} K^{+}(\Omega) h\right]=G(\Omega)+\mathrm{i} \operatorname{sgn}(\sin \Omega) \sqrt{1-G(\Omega)^{2}} \\
& \exp \left[\mathrm{i} K^{-}(\Omega) h\right]=\exp \left[-\mathrm{i} K^{+}(\Omega) h\right]=G(\Omega)-\mathrm{i} \operatorname{sgn}(\sin \Omega) \sqrt{1-G(\Omega)^{2}} .
\end{aligned}
$$

In these expressions, $K^{+}(\Omega)$ and $K^{-}(\Omega)$ can be identified as the wave numbers of the forward-propagating and backward-propagating Bloch waves, respectively. When $|G(\Omega)| \leq 1$, both $K^{+}(\Omega)$ and $K^{-}(\Omega)$ are real and given by 


$$
\cos \left[K^{ \pm}(\Omega) h\right]=G(\Omega), \quad \sin \left[K^{ \pm}(\Omega) h\right]= \pm \operatorname{sgn}(\sin \Omega) \sqrt{1-G(\Omega)^{2}} .
$$

When $|G(\Omega)|>1, K^{+}(\Omega)$ and $K^{-}(\Omega)$ are complex, corresponding to the modes decaying in the positive and negative directions, respectively. Explicitly, $\exp \left[\mathrm{i} K^{+}(\Omega) h\right]$ and $\exp \left[\mathrm{i} K^{-}(\Omega) h\right]$ are real numbers given by

$$
\begin{aligned}
& -1<\exp \left[\mathrm{i} K^{+}(\Omega) h\right]=G(\Omega)+\sqrt{G(\Omega)^{2}-1}<0, \\
& \exp \left[\mathrm{i} K^{-}(\Omega) h\right]=G(\Omega)-\sqrt{G(\Omega)^{2}-1}<-1,
\end{aligned}
$$

when $G(\Omega)<-1$, and

$$
\begin{aligned}
& 0<\exp \left[\mathrm{i} K^{+}(\Omega) h\right]=G(\Omega)-\sqrt{G(\Omega)^{2}-1}<1, \\
& \exp \left[\mathrm{i} K^{-}(\Omega) h\right]=G(\Omega)+\sqrt{G(\Omega)^{2}-1}>1,
\end{aligned}
$$

when $G(\Omega)>1$. The frequency ranges for which $|G(\Omega)| \leq 1$ are the pass bands, and those for which $|G(\Omega)|>1$ are the stop bands, respectively, of the layered structure. From Eqs. (22) and (25b), two eigenvectors of $\mathbf{H}(\Omega)$ can be expressed as

$$
\begin{aligned}
& \mathbf{A}^{+}(\Omega)=\left(\begin{array}{c}
\mathrm{i} \Omega^{-1} \exp (-\mathrm{i} \Omega)\left\{(1-\mathrm{i} \Lambda \Omega) \exp (-\mathrm{i} \Omega)-\exp \left[\mathrm{i} K^{+}(\Omega) h\right]\right\} \\
\Lambda
\end{array}\right), \\
& \mathbf{A}^{-}(\Omega)=\left(\begin{array}{c}
\Lambda \\
-\mathrm{i} \Omega^{-1} \exp (\mathrm{i} \Omega)\left\{(1+\mathrm{i} \Lambda \Omega) \exp (\mathrm{i} \Omega)-\exp \left[-\mathrm{i} K^{+}(\Omega) h\right]\right\}
\end{array}\right),
\end{aligned}
$$

corresponding to the following relations.

$$
\mathbf{H}(\Omega) \mathbf{A}^{+}(\Omega)=\exp \left[\mathrm{i} K^{+}(\Omega) h\right] \mathbf{A}^{+}(\Omega), \quad \mathbf{H}(\Omega) \mathbf{A}^{-}(\Omega)=\exp \left[-\mathrm{i} K^{+}(\Omega) h\right] \mathbf{A}^{-}(\Omega) .
$$

Now, the solution to the problem given by Eqs. (15) and (16) is assumed to be the Bloch mode propagating or decaying in the positive direction of $x$. In this situation, $\mathbf{U}_{1}\left(X_{0+}\right)$ can be expressed as $\mathbf{U}_{1}\left(X_{0+}\right)=C \mathbf{A}^{+}(\Omega)$ where $C$ is a complex constant. Without loss of generality, it can be assumed that $U_{1}\left(X_{0+}\right)=a_{0}$ where $a_{0}>0$ is the characteristic displacement amplitude used for normalization. This condition determines the constant $C$, and the amplitude of the wave is constant in the structure, $\left|U_{1}(x)\right|=a_{0}$, in the pass bands.

Using $\mathbf{U}_{1}\left(X_{m-}\right)=\mathbf{S}(\Omega)^{-1} \mathbf{U}_{1}\left(X_{m+}\right)$ from Eq. (17) and Eq. (30), the displacement gap at the $m$ th interface $x=X_{m}$ is given by

$$
\begin{aligned}
Y_{1 m} & =U_{1 \mathrm{~F}}\left(X_{m+}\right)+U_{1 \mathrm{~B}}\left(X_{m+}\right)-U_{1 \mathrm{~F}}\left(X_{m-}\right)-U_{1 \mathrm{~B}}\left(X_{m-}\right) \\
& =2 \mathrm{i} \Lambda \Omega\left[U_{1 \mathrm{~F}}\left(X_{m+}\right)-U_{1 \mathrm{~B}}\left(X_{m+}\right)\right] \\
& =2 \mathrm{i} \Lambda \Omega \exp \left[\mathrm{i} m K^{+}(\Omega) h\right]\left[U_{1 \mathrm{~F}}\left(X_{0+}\right)-U_{1 \mathrm{~B}}\left(X_{0+}\right)\right] \\
& =2 \mathrm{i} \Lambda W(\Omega) a_{0} \exp \left[\mathrm{i} m K^{+}(\Omega) h\right]
\end{aligned}
$$


where $W(\Omega)$ is defined by

$$
W(\Omega)=\frac{\left\{\exp \left[\mathrm{i} K^{+}(\Omega) h\right]-\exp (-\mathrm{i} \Omega)+2 \Lambda \Omega \sin \Omega\right\} \Omega}{\exp \left[\mathrm{i} K^{+}(\Omega) h\right]-\exp (-\mathrm{i} \Omega)+2 \mathrm{i} \Lambda \Omega \cos \Omega} .
$$

4. Second-harmonic generation in the layered structure

4.1 Governing equations for the second-harmonic generation

The problem of Eqs. (9), (10) and (11b) is rewritten in terms of dimensional variables as

$$
\begin{aligned}
& \frac{\partial^{2} u_{2}}{\partial t^{2}}=c^{2} \frac{\partial^{2} u_{2}}{\partial x^{2}}, X_{m}<x<X_{m+1}, \\
& \rho c^{2} \frac{\partial u_{2}}{\partial x}\left(X_{m+}, t\right)=\rho c^{2} \frac{\partial u_{2}}{\partial x}\left(X_{m-}, t\right)=K_{\mathrm{S}} y_{2 m}(t)-K_{\mathrm{S}} \beta_{m} y_{1 m}(t)^{2},
\end{aligned}
$$

where $u_{2}(x, t)=\varepsilon a_{0} \tilde{u}_{2}(\tilde{x}, \tilde{t})$ and $y_{2 m}(t)=u_{2}\left(X_{m+}, t\right)-u_{2}\left(X_{m-}, t\right)$. This problem is also related to the perfectly periodic layered structure with linear interfacial stiffness, but now excited by the driving forces represented by the second term on the rightmost side of Eq. (34). The driving terms can be obtained from the solution given in the previous section. Substitution of Eq. (31) into Eq. (14b) gives

$$
\begin{aligned}
& y_{1 m}(t)^{2}=2 \Lambda^{2}|W(\Omega)|^{2} a_{0}{ }^{2}\left\{1+\cos \left[2 m K^{+}(\Omega) h+2 \gamma(\Omega)+\pi-2 \omega_{0} t\right]\right\}, \quad|G(\Omega)| \leq 1, \\
& y_{1 m}(t)^{2}=2 \Lambda^{2}|W(\Omega)|^{2} a_{0}{ }^{2} \exp \left[2 \operatorname{im} K^{+}(\Omega) h\right]\left\{1+\cos \left[2 \gamma(\Omega)+\pi-2 \omega_{0} t\right]\right\}, \quad|G(\Omega)|>1,
\end{aligned}
$$

where $\gamma(\Omega)=\operatorname{Arg}[W(\Omega)]$.

The expressions in Eq. (35) clearly indicate that each driving force consists of the DC component $(\omega=0)$ and the double-frequency component $\left(\omega=2 \omega_{0}\right)$. The effect of DC term in the driving force is to superimpose the static displacement field consisting of the gap opening at each interface. Since the present interest is in examining the second-harmonic generation behavior, in what follows the problem is restricted to Eqs. (33)-(35) with the DC terms omitted. Again, the solution to this problem is considered in the frequency domain, introducing

$$
\begin{aligned}
& u_{2}(x, t)=\operatorname{Re}\left[U_{2}(x) \exp \left(-2 \mathrm{i} \omega_{0} t\right)\right], \\
& y_{2 m}(t)=\operatorname{Re}\left[Y_{2 m} \exp \left(-2 \mathrm{i} \omega_{0} t\right)\right],
\end{aligned}
$$

and reducing the problem to

$$
\frac{d^{2} U_{2}}{d x^{2}}+\frac{\left(2 \omega_{0}\right)^{2}}{c^{2}} U_{2}=0, X_{m}<x<X_{m+1},
$$




$$
\rho c^{2} \frac{d U_{2}}{d x}\left(X_{m+}\right)=\rho c^{2} \frac{d U_{2}}{d x}\left(X_{m-}\right)=K_{\mathrm{S}} Y_{2 m}-2 K_{\mathrm{S}} \beta_{m} a_{0}^{2} G_{m},
$$

for the second-harmonic wave field with the double frequency $2 \omega_{0}$, where

$$
G_{m}(\Omega)=-\Lambda^{2} W(\Omega)^{2} \exp \left[2 \mathrm{i} m K^{+}(\Omega) h\right] .
$$

\subsection{Generation and propagation of the second-harmonic component}

The reduced problem given by Eqs. (37)-(39) concerns the second-harmonic wave field excited by the wave sources $-2 K_{\mathrm{S}} \beta_{m} a_{0}{ }^{2} G_{m}$ located at the interfaces $x=X_{m}(m=0, \pm 1, \pm 2, \ldots \ldots)$. In each layer, the displacement components are denoted by $U_{2 \mathrm{~F}}(x)$ and $U_{2 \mathrm{~B}}(x)$ for the forward and backward propagating directions, and the vector with these elements by $\mathbf{U}_{2}(x)=\left(U_{2 \mathrm{~F}}(x), U_{2 \mathrm{~B}}(x)\right)^{\mathrm{T}}$. The propagation characteristics of the Bloch mode at the double frequency are governed by the Bloch wave numbers $K^{+}(2 \Omega)$ and $K^{-}(2 \Omega)$, and their properties are given by Eqs. (24)-(30) with $\Omega$ replaced by $2 \Omega$. The Bloch waves are propagating modes when $|G(2 \Omega)| \leq 1$, and decaying modes when $|G(2 \Omega)|>1$.

An auxiliary problem is first considered which is related to the wave excitation by the single wave source $-2 K_{\mathrm{S}} \beta_{n} a_{0}{ }^{2} G_{n}$ at the interface $x=X_{n}$ alone. With the presence of driving term in Eq. (38) at $x=X_{n}$, the relation between the wave components across the $n$th interface is modified as

$$
\mathbf{U}_{2}\left(X_{n+}\right)=\beta_{n} a_{0}^{2} G_{n} \mathbf{e}+\mathbf{S}(2 \Omega) \mathbf{U}_{2}\left(X_{n-}\right),
$$

instead of Eq. (17), where $\mathbf{e}=(1,1)^{\mathrm{T}}$. The wave field generated by the source at $x=X_{n}$ is restricted to the forward-propagating Bloch mode for $x>X_{n}$ and the backward-propagating one for $x<X_{n}$ from a physical reasoning that it should propagate away from the $n$th interface when $|G(2 \Omega)| \leq 1$ and decay with the distance from it when $|G(2 \Omega)|>1$. Therefore, $\mathbf{U}_{2}\left(X_{n+}\right)$ and $\mathbf{U}_{2}\left(X_{(n-1)+}\right)$ are expressed as

$$
\mathbf{U}_{2}\left(X_{n+}\right)=D^{+} \beta_{n} a_{0}^{2} G_{n} \mathbf{A}^{+}(2 \Omega), \quad \mathbf{U}_{2}\left(X_{(n-1)+}\right)=D^{-} \beta_{n} a_{0}^{2} G_{n} \mathbf{A}^{-}(2 \Omega),
$$

where $D^{+}$and $D^{-}$are complex constants. Substitution of Eq. (41) in Eq. (40) gives

$$
D^{+} \mathbf{A}^{+}(2 \Omega)-D^{-} \exp \left[-\mathrm{i} K^{+}(2 \Omega) h\right] \mathbf{A}^{-}(2 \Omega)=\mathbf{e},
$$

which in turn determines two constants as

$$
D^{+}=-\frac{2 \mathrm{i} \Omega \exp (2 \mathrm{i} \Omega)\left\{\exp (2 \mathrm{i} \Omega)-\exp \left[-\mathrm{i} K^{+}(2 \Omega) h\right]-4 \Lambda \Omega \sin (2 \Omega)\right\}}{2-(1+2 \mathrm{i} \Lambda \Omega) \exp (2 \mathrm{i} \Omega) \exp \left[\mathrm{i} K^{+}(2 \Omega) h\right]-(1-2 \mathrm{i} \Lambda \Omega) \exp (-2 \mathrm{i} \Omega) \exp \left[-\mathrm{i} K^{+}(2 \Omega) h\right]},
$$




$$
\begin{aligned}
& D^{-} \exp \left[-\mathrm{i} K^{+}(2 \Omega) h\right]= \\
& -\frac{2 \mathrm{i} \Omega \exp (-2 \mathrm{i} \Omega)\left\{\exp (-2 \mathrm{i} \Omega)-\exp \left[\mathrm{i} K^{+}(2 \Omega) h\right]-4 \Lambda \Omega \sin (2 \Omega)\right\}}{2-(1+2 \mathrm{i} \Lambda \Omega) \exp (2 \mathrm{i} \Omega) \exp \left[\mathrm{i} K^{+}(2 \Omega) h\right]-(1-2 \mathrm{i} \Lambda \Omega) \exp (-2 \mathrm{i} \Omega) \exp \left[-\mathrm{i} K^{+}(2 \Omega) h\right]}
\end{aligned}
$$

Once $\mathbf{U}_{2}\left(X_{n+}\right)$ and $\mathbf{U}_{2}\left(X_{(n-1)+}\right)$ are determined by Eqs. (41) and (43), the wave vector at $x=X_{m+}$ is given by

$$
\begin{aligned}
\mathbf{U}_{2}\left(X_{m+}\right) & =\mathbf{H}(2 \Omega)^{m-n} \mathbf{U}_{2}\left(X_{n+}\right) \\
& =-\Lambda^{2} W(\Omega)^{2} \beta_{n} a_{0}{ }^{2} \exp \left[2 \mathrm{i} n K^{+}(\Omega) h\right] \exp \left[\mathrm{i}(m-n) K^{+}(2 \Omega) h\right] \mathbf{E}^{+}(2 \Omega)
\end{aligned}
$$

for $m \geq n$, and

$$
\begin{aligned}
\mathbf{U}_{2}\left(X_{m+}\right) & =\mathbf{H}(2 \Omega)^{-(n-m-1)} \mathbf{U}_{2}\left(X_{(n-1)+}\right) \\
& =\Lambda^{2} W(\Omega)^{2} \beta_{n} a_{0}{ }^{2} \exp \left[2 \mathrm{i} n K^{+}(\Omega) h\right] \exp \left[-\mathrm{i}(m-n) K^{+}(2 \Omega) h\right] \mathbf{E}^{-}(2 \Omega)
\end{aligned}
$$

for $m \leq n-1$, using Eq. (39) and introducing

$$
\mathbf{E}^{+}(2 \Omega)=D^{+} \mathbf{A}^{+}(2 \Omega), \mathbf{E}^{-}(2 \Omega)=-D^{-} \exp \left[-\mathrm{i} K^{+}(2 \Omega) h\right] \mathbf{A}^{-}(2 \Omega) .
$$

By superposing the above auxiliary solution for different $n$, the solution to the original problem involving nonlinear interfaces at $x=X_{n}(n=0, \pm 1, \pm 2, \ldots \ldots)$ is formally expressed as

$$
\begin{aligned}
\mathbf{U}_{2}\left(X_{m+}\right)= & \sum_{n=-\infty}^{m} D^{+} \beta_{n} a_{0}{ }^{2} G_{n} \mathbf{H}(2 \Omega)^{m-n} \mathbf{A}^{+}(2 \Omega)+\sum_{n=m+1}^{\infty} D^{-} \beta_{n} a_{0}{ }^{2} G_{n} \mathbf{H}(2 \Omega)^{-(n-m-1)} \mathbf{A}^{-}(2 \Omega) \\
=-\Lambda^{2} W(\Omega)^{2} a_{0}{ }^{2}\{ & \exp \left[\mathrm{im} K^{+}(2 \Omega) h\right] \mathbf{E}^{+}(2 \Omega) \sum_{n=-\infty}^{m} \beta_{n} \exp [\mathrm{i} n \Delta K(\Omega) h] \\
& \left.-\exp \left[-\mathrm{i} m K^{+}(2 \Omega) h\right] \mathbf{E}^{-}(2 \Omega) \sum_{n=m+1}^{\infty} \beta_{n} \exp [4 \mathrm{i} n \bar{K}(\Omega) h]\right\},
\end{aligned}
$$

where

$$
\Delta K(\Omega)=2 K^{+}(\Omega)-K^{+}(2 \Omega), \bar{K}(\Omega)=\frac{1}{4}\left[2 K^{+}(\Omega)+K^{+}(2 \Omega)\right] .
$$

The above expressions indicate that the field of the second-harmonic component is influenced by the Bloch wave propagation characteristics at $\omega=\omega_{0}$ as well as $\omega=2 \omega_{0}$. This issue is examined further below for two special cases.

\section{Results and discussions for special cases}

5.1 Second-harmonic generation by a single nonlinear interface

When all the interfaces are linear except one at $x=X_{n} \geq X_{0}$, i.e., $\beta_{m}=0$ when $m \leq n-1$ and $m \geq n+1$, the second-harmonic field is given by Eqs. $(44 \mathrm{a}, \mathrm{b})$ with $\beta_{n}=\beta$ where $\beta$ is a positive constant. Let us consider the second-harmonic amplitude at a fixed location in the layered structure, 
say at $x=X_{m^{+}}>X_{n}$ as shown schematically in Fig. 2. As clearly shown in Eq. (44a), the second-harmonic amplitude at $x=X_{m+}$ is governed by several factors. First, it depends on the distance of the location of the nonlinear interface $\left(x=X_{n}\right)$ from the reference position $\left(x=X_{0}\right)$ as represented by the factor $\left|\exp \left[2 \mathrm{i} n K^{+}(\Omega) h\right]\right|$. This factor is related to the reduction of the amplitude of the fundamental component at the nonlinear interface when $|G(\Omega)|>1$, i.e., when the fundamental frequency is in one of the stop bands of the layered structure. Second, $|W(\Omega)|$ is related to the magnitude of gap opening of the interface. From Eq. (32), $|W(\Omega)|$ becomes zero at the left edge of each pass band, $\Omega=k \pi(k=0,1,2, \ldots \ldots)$, thus forbids the second-harmonic generation. On the other hand, at the frequencies satisfying $\tan \Omega=-2 \Lambda \Omega$ which lie in the stop bands, $|W(\Omega)|$ becomes unbounded. Third, the factor $\left|E^{+}(2 \Omega)\right|$, where $E^{+}(2 \Omega) \equiv \mathbf{E}^{+}(2 \Omega) \cdot \mathbf{e}$, determines the generation efficiency of the second-harmonic component at the nonlinear interface. From Eqs. (29a) and (43a), this quantity becomes zero at the frequency satisfying $\tan (2 \Omega)=-4 \Lambda \Omega$, while it diverges to infinity when the double frequency is at the right edge of each pass band. Finally, when $|G(2 \Omega)|>1$, the double frequency is in one of the stop bands, and the second-harmonic component suffers damping by the factor $\left|\exp \left[\mathrm{i}(m-n) K^{+}(2 \Omega) h\right]\right|$ depending on the distance between the observation point $x=X_{m+}$ and the nonlinear interface at $x=X_{n}$. When $\Lambda=0.2$, the factors $\left|\exp \left[2 \mathrm{i} i K^{+}(\Omega) h\right]\right|$ and $\left|\exp \left[\mathrm{i}(m-n) K^{+}(2 \Omega) h\right]\right|$ for $m=10$ and two different values of $n(n=1$ and $n=9)$ are shown in Fig. 3 as functions of the normalized fundamental frequency together with the factors $|W(\Omega)|$ and $\left|E^{+}(2 \Omega)\right|$.

The frequency dependence of the second-harmonic amplitude at $x=X_{10+}(m=10)$ is shown in Fig. 4 when the nonlinear interface is at (a) $x=X_{1} \quad(n=1)$ and (b) $x=X_{9} \quad(n=9)$. The dispersion curves $K^{+}(\Omega)$ and $K^{+}(2 \Omega)$ are also shown in Fig. 4 in order to ease the comparison of the second-harmonic amplitude spectrum and the band structure. In Fig. 4 (a) for which the nonlinear interface is at $x=X_{1}$, the second-harmonic amplitude is significantly influenced by the factor $\left|\exp \left[\mathrm{i}(m-n) K^{+}(2 \Omega) h\right]\right|$ while it is less influenced by $\left|\exp \left[2 \mathrm{i} n K^{+}(\Omega) h\right]\right|$ as the nonlinear interface is relatively close to $x=X_{0}$. As a consequence, the observed second-harmonic component is almost zero when the double frequency is in one of the stop bands. In Fig. 4 (b) for $n=9$, on the contrary, the second-harmonic amplitude is influenced by $\left|\exp \left[2 \mathrm{in} K^{+}(\Omega) h\right]\right|$ but less so by $\left|\exp \left[\mathrm{i}(m-n) K^{+}(2 \Omega) h\right]\right|$. Therefore the second-harmonic amplitude is almost zero when the fundamental frequency is in one of the stop bands. Within the stop bands of the fundamental frequency, however, there are sharp peaks of the second-harmonic amplitude due to the diverging nature of 
$\left|E^{+}(2 \Omega)\right|$ when the double frequency is at the right edge of the pass bands. It is noted, however, that the perturbation analysis loses its validity at the frequencies where $\left|U_{2}\right|$ shows diverging behavior.

\subsection{Second-harmonic generation by multiple consecutive nonlinear interfaces}

Consider next the case where multiple consecutive interfaces have identical nonlinearity as shown in Fig. 5. When the nonlinearity parameters take

$$
\beta_{n}=\left\{\begin{array}{cc}
0, & n \leq 0, \quad n \geq N+1, \\
\beta, & 1 \leq n \leq N,
\end{array}\right.
$$

where $N$ is a positive integer representing the number of nonlinear interfaces, Eq. (46) reduces to

$$
\begin{gathered}
\mathbf{U}_{2}\left(X_{m+}\right)=\Lambda^{2} W(\Omega)^{2} \beta a_{0}{ }^{2} F(4 \bar{K}(\Omega) h, N) \exp \left[-\mathrm{i} m K^{+}(2 \Omega) h\right] \mathbf{E}^{-}(2 \Omega), \quad m \leq 0, \\
\mathbf{U}_{2}\left(X_{m+}\right)=-\Lambda^{2} W(\Omega)^{2} \beta a_{0}{ }^{2}\left\{F(\Delta K(\Omega) h, m) \exp \left[i m K^{+}(2 \Omega) h\right] \mathbf{E}^{+}(2 \Omega)\right. \\
\left.\quad-F(4 \bar{K}(\Omega) h, N-m) \exp \left[2 \mathrm{i} m K^{+}(\Omega) h\right] \mathbf{E}^{-}(2 \Omega)\right\}, \quad 1 \leq m \leq N, \\
\mathbf{U}_{2}\left(X_{m+}\right)=-\Lambda^{2} W(\Omega)^{2} \beta a_{0}^{2} F(\Delta K(\Omega) h, N) \exp \left[i m K^{+}(2 \Omega) h\right] \mathbf{E}^{+}(2 \Omega), \quad m \geq N+1,
\end{gathered}
$$

where the function $F(\xi, M)$ is defined by

$$
F(\xi, M) \equiv\left\{\begin{array}{cc}
\frac{1-\exp (\mathrm{i} M \xi)}{\exp (-\mathrm{i} \xi)-1}, & \exp (\mathrm{i} \xi) \neq 1, \\
M, & \exp (\mathrm{i} \xi)=1 .
\end{array}\right.
$$

The expression in Eq. (49b) shows that the amplitudes of the forward and backward Bloch waves oscillate with $m$ for $1 \leq m \leq N$. The wavelengths of oscillations are governed by $\Delta K(\Omega)$ for the forward wave and by $4 \bar{K}(\Omega)$ for the backward one. Likewise, in Eq. (49c) the second-harmonic amplitude for $m \geq N+1$ oscillates with the number of nonlinear interfaces $N$. The exception arises when $\exp [\mathrm{i} \Delta K(\Omega) h]=1$ corresponding to the so-called phase matching between the fundamental and second-harmonic Bloch waves. If this condition is met when the fundamental and the double frequencies are both in the pass bands of the structure, the second-harmonic Bloch waves have the same phase velocity with the fundamental ones, and the second-harmonic component is generated in a cumulative manner. Namely, the amplitude of the forward-propagating second-harmonic Bloch wave increases in proportion with the distance in the layered structure with nonlinear interfaces. From Eqs. (24) and (25), however, the exact phase matching occurs only at $\Omega=k \pi,(k=0,1,2, \ldots \ldots)$, which correspond to the left edge of the pass bands of the structure. At these frequencies, the second-harmonic amplitude is zero since $W(k \pi)=0$. It is then only expected that the second-harmonic component grows nearly proportionally with the distance at frequencies near $\Omega=k \pi$ in each pass band. 
In the cases when the number of nonlinear interfaces is $N=10,20,30$ and 40, the amplitude of the second-harmonic component at $x=X_{40+}$ is shown in Fig. 6 as a function of the normalized fundamental frequency for different values of $\Lambda$. In Fig. 6 , it is first seen that the second-harmonic amplitude exhibits sharp peaks when the double frequency is at the right edge of the pass bands. This is due to the diverging nature of $\left|E^{+}(2 \Omega)\right|$ as discussed in the previous section for a single nonlinear interface. Furthermore, the diverging behavior of $|W(\Omega)|$ also contributes to enhance the second-harmonic amplitude when the double frequency is in one of the pass bands even if the fundamental one is in the stop bands.

The band structure of the layered structure with spring-type interfaces is governed by the non-dimensional parameter $\Lambda$ [21]. When $\Lambda \rightarrow 0$, the interfaces become perfectly bonded and the dispersive nature disappears. When $\Lambda$ is sufficiently small, the dispersion is weak and $\Delta K(\Omega)$ remains small except near the right edge of each pass band. As a consequence, in Fig. 6 (a) for $\Lambda=$ 0.02 the second-harmonic amplitude tends to grow nearly cumulatively in each pass band, thus proportionally increasing with $N$. As $\Lambda$ increases, however, each pass band becomes narrower and the dispersive nature more remarkable. In Fig. 6 (d) for $\Lambda=0.4$, monotonic growth of the second-harmonic amplitude is only seen near $\Omega=0$ and $\Omega=\pi$.

In the case when $\Lambda=0.2$ and $N=30$, the distribution of the second-harmonic amplitude in the layered structure is shown in Fig. 7 for different normalized fundamental frequencies. Note that the amplitude exhibits stepwise variation across the interfaces and remains constant in each layer, since the layers are linearly elastic. For $\Omega / \pi=0.1$ and 0.3 , both the fundamental and the double frequencies are in the first pass band. As a consequence, the second-harmonic amplitude increases cumulatively with distance for the region $0<x / h<30$ in which the nonlinear interfaces lie. For $\Omega / \pi=0.4$, the fundamental component can propagate through the structure and generate the second-harmonic component at 30 interfaces, but the second-harmonic field has a standing-wave nature and does not propagate away to infinity since the double frequency is in the first stop band. For $\Omega / \pi=0.5$ and 0.6 , the fundamental and the double frequencies are in the first and the second pass bands, respectively, but due to the dispersive nature the second-harmonic amplitude oscillates with certain wavelengths mainly governed by $\Delta K(\Omega)$. For $\Omega / \pi=0.8$, these frequencies are in the first and the second stop bands, respectively, so the fundamental component decays for $x / h>0$, which only accompanies the second-harmonic generation in a localized nature. For $\Omega / \pi=1.1$, both frequencies are in the pass bands and the cumulative nature of second-harmonic generation is recovered due to the approximate phase matching near $\Omega=\pi$.

\subsection{Low-frequency approximation}


When the fundamental frequency is sufficiently low, the dispersion relation of the Bloch wave is approximated to the first order of $\Omega$ as

$$
K^{+}(\Omega) h \approx \sqrt{1+2 \Lambda} \Omega,
$$

from Eqs. (24) and (26). Likewise, the quantities introduced in the above derivation can be approximated as follows by retaining the lowest-order terms of $\Omega$.

$$
\begin{aligned}
& W(\Omega) \approx \frac{\Omega}{\sqrt{1+2 \Lambda}}, \\
& \mathbf{E}^{+}(2 \Omega) \approx \frac{1}{1+2 \Lambda+\sqrt{1+2 \Lambda}}\left(\begin{array}{c}
1+\Lambda+\sqrt{1+2 \Lambda} \\
\Lambda
\end{array}\right), \\
& \mathbf{E}^{-}(2 \Omega) \approx \frac{1}{1+2 \Lambda+\sqrt{1+2 \Lambda}}\left(\begin{array}{c}
\Lambda \\
1+\Lambda+\sqrt{1+2 \Lambda}
\end{array}\right) .
\end{aligned}
$$

As a consequence, in the first case of a single nonlinear interface discussed above, Eqs. (44a, b) reduce to

$$
\begin{aligned}
& U_{2}\left(X_{m+}\right)=\frac{\Lambda^{2}}{1+2 \Lambda} \Omega^{2} \beta a_{0}{ }^{2} \exp [2 \mathrm{i}(2 n-m) \sqrt{1+2 \Lambda} \Omega], \quad m \leq n-1, \\
& U_{2}\left(X_{m+}\right)=-\frac{\Lambda^{2}}{1+2 \Lambda} \Omega^{2} \beta a_{0}{ }^{2} \exp (2 \mathrm{i} m \sqrt{1+2 \Lambda} \Omega), \quad m \geq n,
\end{aligned}
$$

which make clear that the second-harmonic amplitude in the layered structure is $\left[\Lambda^{2} /(1+2 \Lambda)\right] \beta a_{0}^{2} \Omega^{2}$, exhibiting quadratic frequency dependence.

In the second case of $N$ consecutive nonlinear interfaces, the displacement at $x=X_{m+}$ is given by

$$
\begin{aligned}
U_{2}\left(X_{m+}\right) & =\mathrm{i} \frac{\Lambda^{2} \Omega \beta a_{0}^{2}}{4(1+2 \Lambda)^{3 / 2}}[1-\exp (4 \mathrm{i} N \sqrt{1+2 \Lambda} \Omega)] \exp (-2 \mathrm{i} m \sqrt{1+2 \Lambda} \Omega), m \leq 0, \quad \text { (55a) } \\
U_{2}\left(X_{m+}\right) & =-\frac{\Lambda^{2} \Omega^{2} \beta a_{0}^{2}}{1+2 \Lambda} m \exp (2 \mathrm{i} m \sqrt{1+2 \Lambda} \Omega) \\
& +\mathrm{i} \frac{\Lambda^{2} \Omega \beta a_{0}^{2}}{4(1+2 \Lambda)^{3 / 2}}\{\exp (2 \mathrm{i} m \sqrt{1+2 \Lambda} \Omega)-\exp [2(2 N-m) \mathrm{i} \sqrt{1+2 \Lambda} \Omega]\}, \quad 1 \leq m \leq N \\
U_{2}\left(X_{m+}\right) & =-\frac{\Lambda^{2} \Omega^{2} \beta a_{0}^{2}}{1+2 \Lambda} N \exp (2 \mathrm{i} m \sqrt{1+2 \Lambda} \Omega), m \geq N+1
\end{aligned}
$$

The first term on the right-hand side of Eq. (55b) shows that the forward-propagating second-harmonic component is generated cumulatively with quadratic frequency dependence. As shown in Eq. (55c), the second-harmonic amplitude beyond the $N$ interfaces is given by 
$\left[\Lambda^{2} /(1+2 \Lambda)\right] N \beta a_{0}{ }^{2} \Omega^{2}$ which is exactly $N$ times that for a single nonlinear interface. These features are due to the approximation in Eq. (51) which means that the structure is now non-dispersive. In Fig. 8, the low-frequency parts of Fig. 6 (a) and (c) are redrawn in the form scaled as $\left|U_{2}\right| /\left(N \beta a_{0}{ }^{2}\right)$. The low-frequency formula $\left|U_{2}\right| /\left(N \beta a_{0}{ }^{2}\right)=\left[\Lambda^{2} /(1+2 \Lambda)\right] \Omega^{2}$ is also shown in Fig. 8. In Fig. 8 (a) for $\Lambda$ $=0.02$, the low-frequency approximation gives excellent agreement with the exact results until the double frequency enters the first stop band when $\Omega / \pi \cong 0.5$. In Fig. 8 (b) for $\Lambda=0.2$, however, the agreement is limited to a lower frequency range, say $\Omega / \pi<0.2$, due to the more remarkable dispersive nature.

\section{Conclusion}

The second-harmonic generation characteristics in the longitudinal wave propagation across an infinite layered structure consisting of linear elastic layers and nonlinear spring-type interfaces have been analyzed theoretically using a perturbation approach. An explicit analytical expression has been derived for the second-harmonic field in the layered structure. The analysis has revealed that the second-harmonic generation behavior depends significantly on the fundamental frequency reflecting the band structure of the layered structure. Two special cases have been discussed in order to clarify such dependence, i.e., the second-harmonic generation by a single as well as multiple consecutive nonlinear interfaces. In particular, when the second-harmonic generation occurs at multiple consecutive nonlinear interfaces, the cumulative growth of the second-harmonic amplitude with distance is only expected in certain frequency ranges where the fundamental as well as the double frequencies belong to the pass bands. Furthermore, a remarkable increase of the second-harmonic amplitude has been found near the terminating edge of pass bands. Approximate expressions for the second-harmonic amplitude have been obtained when the frequency is sufficiently low, which show that the second-harmonic generation occurs cumulatively with quadratic frequency dependence.

The present analysis assumed an infinite layered structure of identical linear elastic layers and interlayer interfaces with identical linear stiffness, in order to obtain explicit analytical expressions for the second-harmonic amplitude. The perturbation approach presented here can, however, be applied to analyze the second-harmonic generation behavior in more general cases such as structures consisting of dissimilar elastic layers and interfacial stiffnesses, or finitely extended layered structures.

\section{Acknowledgments}

This work has been supported by JSPS KAKENHI Grant Numbers 15K13833 and 25-1754.

\section{References}

[1] L. J. Pyrak-Nolte, The seismic response of fractures and the interrelations among fracture 
properties, Int. J. Rock Mech. Min. Sci. \& Geomech. Abstr. 33 (1996) 787-802.

[2] A. M. Quinn, B. W. Drinkwater, R. S. Dwyer-Joyce, The measurement of contact pressure in machine elements using ultrasound, Ultrasonics 39 (2002) 495-502.

[3] R. S. Dwyer-Joyce, The application of ultrasonic NDT techniques in tribology, Proc. IMechE J. Eng. Trib. 219 (2005) 347-366.

[4] C. J. Brotherhood, B. W. Drinkwater, S. Dixon, The detectability of kissing bonds in adhesive joints using ultrasonic techniques, Ultrasonics 41 (2003) 521-529.

[5] M. A. S. Akanda, M. Saka, Relationship between closure stress of small fatigue crack and ultrasonic response, J. Nondestruct. Eval. 23 (2004) 37-47.

[6] J.-Y. Kim, V. A. Yakovlev, S. I. Rokhlin, Surface acoustic wave modulation on a partially closed fatigue crack, J. Acoust. Soc. Am. 115 (2004) 1961-1972.

[7] K. Kendall, D. Tabor, An ultrasonic study of the area of contact between stationary and sliding surfaces, Proc. R. Soc. Lond. A323 (1971) 321-340.

[8] J.-M. Baik, R. B. Thompson, Ultrasonic scattering from imperfect interfaces: a quasi-static model, J. Nondestruct. Eval. 4 (1984) 177-196.

[9] S. I. Rokhlin, Y. J. Wang, Analysis of boundary conditions for elastic wave interaction with an interface between two solids, J. Acoust. Soc. Am. 89 (1991) 503-515.

[10] P. B. Nagy, Ultrasonic classification of imperfect interfaces, J. Nondestruct. Eval. 11 (1992) 127-139.

[11] B. W. Drinkwater, R. S. Dwyer-Joyce, P. Cawley, A study of the interaction between ultrasound and a partially contacting solid-solid interface, Proc. R. Soc. Lond. A452 (1996) 2613-2628.

[12] A. Boström, G. Wickham, On the boundary conditions for ultrasonic transmission by partially closed cracks, J. Nondestruct. Eval. 10 (1991) 139-149.

[13] C. Pecorari, Scattering of a Rayleigh wave by a surface-breaking crack with faces in partial contact, Wave Motion 33 (2001) 259-270.

[14] J. P. Jones, J. S. Whittier, Waves at a flexibly bonded interface, Trans. ASME J. Appl. Mech. (1967) 905-909.

[15] L. J. Pyrak-Nolte, N. G. W. Cook, Elastic interface waves along a fracture, Geophys. Res. Lett. 14 (1987) 1107-1110.

[16] B. Gu, K. T. Nihei, L. R. Myer, L. J. Pyrak-Nolte, Fracture interface waves, J. Geophys. Res. 101 (1996) 827-835.

[17] S. Biwa, S. Hiraiwa, E. Matsumoto, Stiffness evaluation of contacting surfaces by bulk and interface waves, Ultrasonics 47 (2007) 123-129.

[18] Y. Lu, J. D. Achenbach, Effects of random deviations in interface properties on the propagation of ultrasound in thick composites, J. Acoust. Soc. Am. 90 (1991) 2576-2585.

[19] Y. Lu, Effects of random fluctuations in ply mechanical properties on ultrasound propagation in a laminated solid layer, Ultrasonics 30 (1992) 289-295. 
[20] L. Wang, S. I. Rokhlin, Ultrasonic wave interaction with multidirectional composites: modeling and experiment, J. Acoust. Soc. Am. 114 (2003) 2582-2895.

[21] Y. Ishii, S. Biwa, Ultrasonic evaluation of interlayer interfacial stiffness of multilayered structures, J. Appl. Phys. 111 (2012) 084907.

[22] Y. Ishii, S. Biwa, Evaluation of interlayer interfacial stiffness and layer wave velocity of multilayered structures by ultrasonic spectroscopy, J. Acoust. Soc. Am. 136 (2014) 183-191.

[23] Y. Ishii, S. Biwa, Transmission of ultrasonic waves at oblique incidence to composite laminates with spring-type interlayer interfaces, submitted for publication.

[24] A. M. Sutin, V. E. Nazarov, Nonlinear acoustic methods of crack diagnosis, Radiophys. Quant. Electr. 38 (1995) 109-120.

[25] I. Y. Solodov, Ultrasonics of non-linear contacts: propagation, reflection and NDE-applications, Ultrasonics 36 (1998) 383-390.

[26] O. Buck, W. L. Morris, J. M. Richardson, Acoustic harmonic generation at unbonded interfaces and fatigue cracks, Appl. Phys. Lett. 33 (1978) 371-373.

[27] J. M. Richardson, Harmonic generation at an unbonded interface, I. Planar interface between semi-infinite elastic media, Int. J. Engng Sci. 17 (1979) 73-85.

[28] S. Hirose, J. D. Achenbach, Higher harmonics in the far field due to dynamic crack-face contacting, J. Acoust. Soc. Am. 93 (1993) 142-147.

[29] C. Pecorari, Nonlinear interaction of plane ultrasonic waves with an interface between rough surfaces in contact, J. Acoust. Soc. Am. 113 (2003) 3065-3072.

[30] S. Biwa, S. Nakajima, N. Ohno, On the acoustic nonlinearity of solid-solid contact with pressure-dependent interface stiffness, Trans. ASME J. Appl. Mech. 71 (2004) 508-515.

[31] C. Pecorari, M. Poznic, On the linear and nonlinear acoustic properties of dry and water-confining elasto-plastic interfaces, Proc. R. Soc. A462 (2006) 769-788.

[32] S. Biwa, S. Hiraiwa, E. Matsumoto, Experimental and theoretical study of harmonic generation at contacting interface, Ultrasonics 44 (2006) e1319-e1322.

[33] J.-Y. Kim, A. Baltazar, J. W. Hu, S. I. Rokhlin, Hysteretic linear and nonlinear acoustic responses from pressed interfaces, Int. J. Solids Struct. 43 (2006) 6436-6452.

[34] J.-Y. Kim, J.-S. Lee, A micromechanical model for nonlinear acoustic properties of interfaces between solids, J. Appl. Phys. 101 (2007) 043501.

[35] D. Yan, B. W. Drinkwater, S. A. Neild, Measurement of the ultrasonic nonlinearity of kissing bonds in adhesive joints, NDT\&E Int. 42 (2009) 459-466.

[36] D. Yan, S. A. Neild, B. W. Drinkwater, Modelling and measurement of the nonlinear behavior of kissing bonds in adhesive joints, NDT\&E Int. 47 (2012) 18-25.

[37] Y. Ishii, S. Biwa, Acoustic harmonic generation in a multilayered structure with nonlinear interfaces, Nonlinear Acoustics, State-of-the-Art and Perspectives: 19th International Symposium on Nonlinear Acoustics (eds. T. Kamakura and N. Sugimoto); AIP Conference Proceedings 1474 
(2012) 223-226.

[38] K. Manktelow, R. K. Narisetti, M. J. Leamy, M. Ruzzene, Finite-element based perturbation analysis of wave propagation in nonlinear periodic structures, Mech. Sys. Signal Process. 39 (2013) 32-46.

[39] K. Manktelow, M. J. Leamy, M. Ruzzene, Comparison of asymptotic and transfer matrix approaches for evaluating intensity-dependent dispersion in nonlinear photonic and phononic crystals, Wave Motion 50 (2013) 494-508.

[40] A. F. Vakakis, M. E. King, Resonant oscillations of a weakly coupled, nonlinear layered system, Acta Mech. 128 (1998) 59-80.

[41] A. V. Porubov, I. V. Andrianov, V. V. Danishevs'kyy, Nonlinear strain wave localization in periodic composites, Int. J. Solids Struct. 49 (2012) 3381-3387.

[42] I. V. Andrianov, V. V. Danishevs'kyy, O. I. Ryzhkov, D. Weichert, Dynamic homogenization and wave propagation in a nonlinear 1D composite material, Wave Motion 50 (2013) 271-281.

[43] Y. Yun, G. Q. Miao, P. Zhang, K. Huang, R. J. Wei, Nonlinear acoustic wave propagating in one-dimensional layered system, Phys. Lett. A 343 (2005) 351-358.

[44] U. Polimeno, M. Meo, Detecting barely visible impact damage detection on aircraft composites structures, Compos. Struct. 91 (2009) 398-402.

[45] S. Delrue, K. Van Den Abeele, Three-dimensional finite element simulation of closed delaminations in composite materials, Ultrasonics 52 (2012) 315-324.

[46] L. A. Ostrovsky, P. A. Johnson, Dynamic nonlinear elasticity in geomaterials, Rivista Del Nuovo Cimento 24 (2001) 1-46.

[47] J. Cabaret, V. Tournat, P. Béquin, Amplitude-dependent phononic processes in a diatomic granular chain in the weakly nonlinear regime, Phys. Rev. E 86 (2012) 041305.

[48] W. T. Thomson, Transmission of elastic waves through a stratified solid medium, J. Appl. Phys. 21 (1950) 89-93.

[49] N. A. Haskell, The dispersion of surface waves on multilayered media, Bull. Seismol. Soc. Am. $43(1953)$ 17-34. 
Figure legends

Fig. 1 A periodic layered structure consisting of linear elastic layers and spring-type interlayer interfaces.

Fig. 2 A schematic for the second-harmonic generation by a single nonlinear spring-type interface in the layered structure.

Fig. 3 Frequency dependence of four factors governing the second-harmonic amplitude observed in the layered structure.

Fig. 4 Frequency dependence of the second-harmonic amplitude at $x=X_{10+}$ when $\Lambda=0.2$ and the nonlinear interface is located at (a) $x=X_{1}$ and (b) $x=X_{9}$. The dispersion curves of the layered structure are also shown.

Fig. 5 A schematic for the second-harmonic generation by multiple consecutive nonlinear interfaces in the layered structure.

Fig. 6 Frequency dependence of the second-harmonic amplitude at $x=X_{40+}$ for different numbers of nonlinear interfaces $(N=10,20,30,40)$ when (a) $\Lambda=0.02$, (b) $\Lambda=0.1$, (c) $\Lambda=0.2$ and (d) $\Lambda=0.4$. Note that the vertical scale for (a) is different from the others.

Fig. 7 The second-harmonic amplitude distribution in the layered structure for $\Lambda=0.2$ and different normalized frequencies $\Omega$. Note that the vertical scales are not the same.

Fig. 8 Frequency dependence of the second-harmonic amplitude at $x=X_{40+}$ for different numbers of nonlinear interfaces $(N=10,20,30,40)$ when (a) $\Lambda=0.02$ and (b) $\Lambda=0.2$, together with the low-frequency approximation. Note that the vertical scales for (a) and (b) are not the same. 


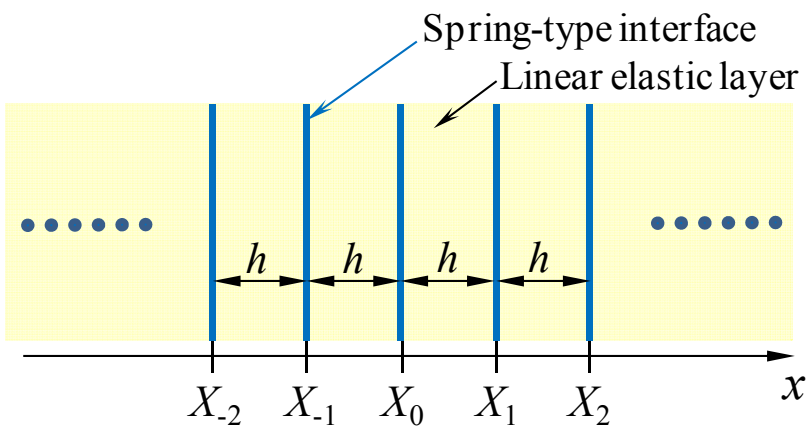

Fig. 1 A periodic layered structure consisting of linear elastic layers and spring-type interlayer interfaces.

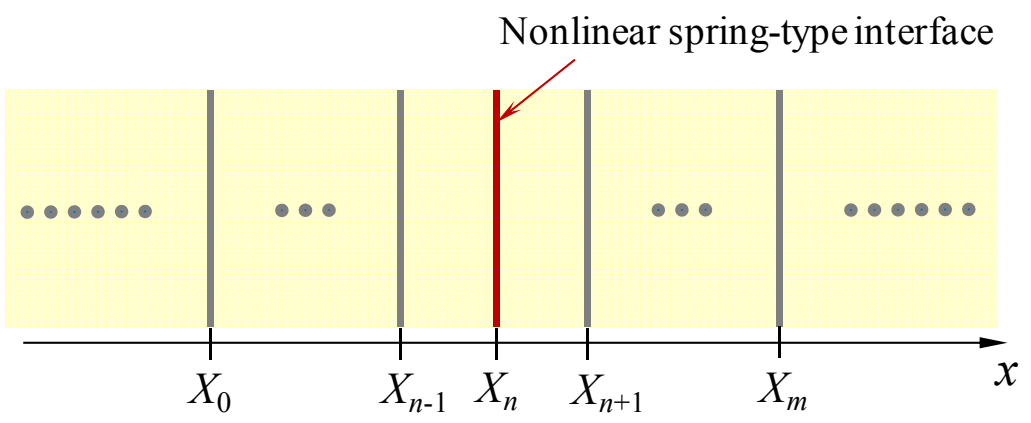

Fig. 2 A schematic for the second-harmonic generation by a single nonlinear spring-type interface in the layered structure. 

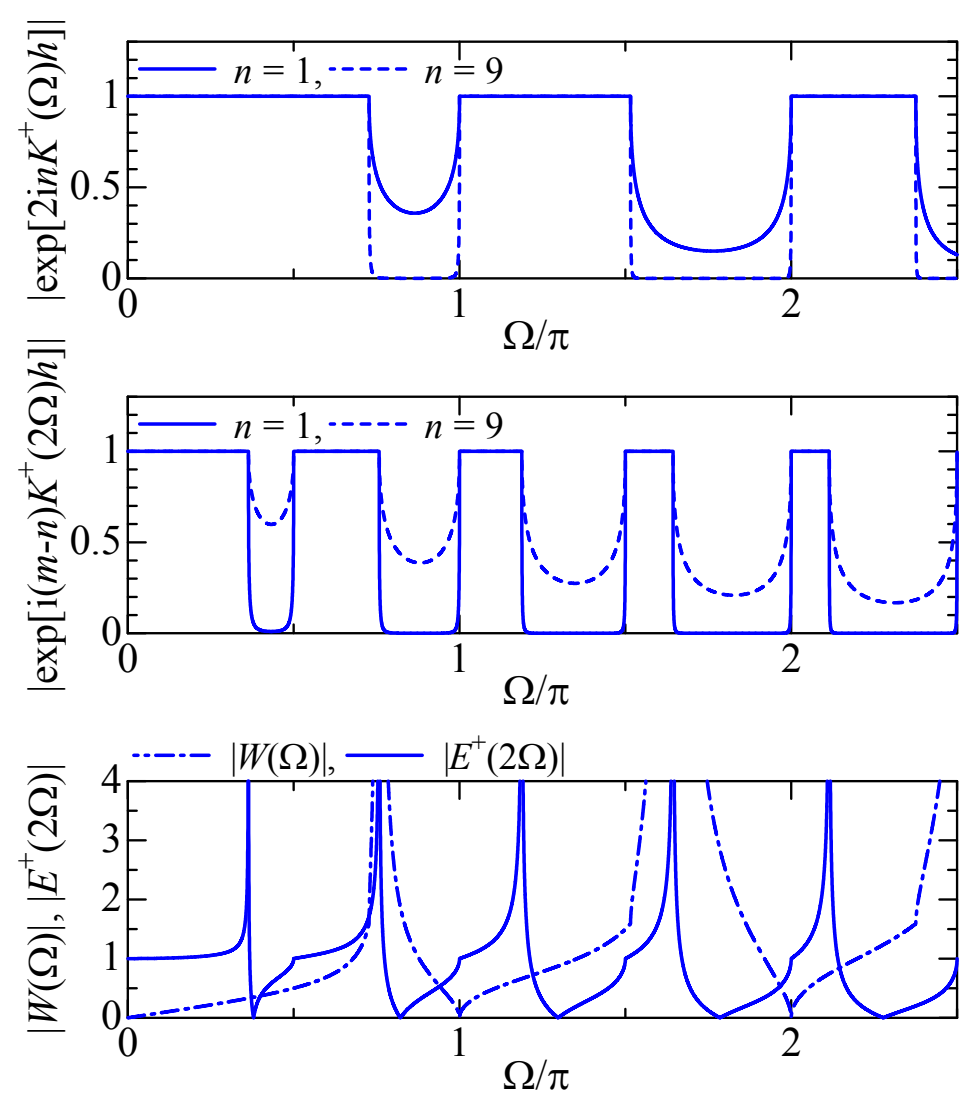

Fig. 3 Frequency dependence of four factors governing the second-harmonic amplitude observed in the layered structure. 
(a) $n=1, m=10$

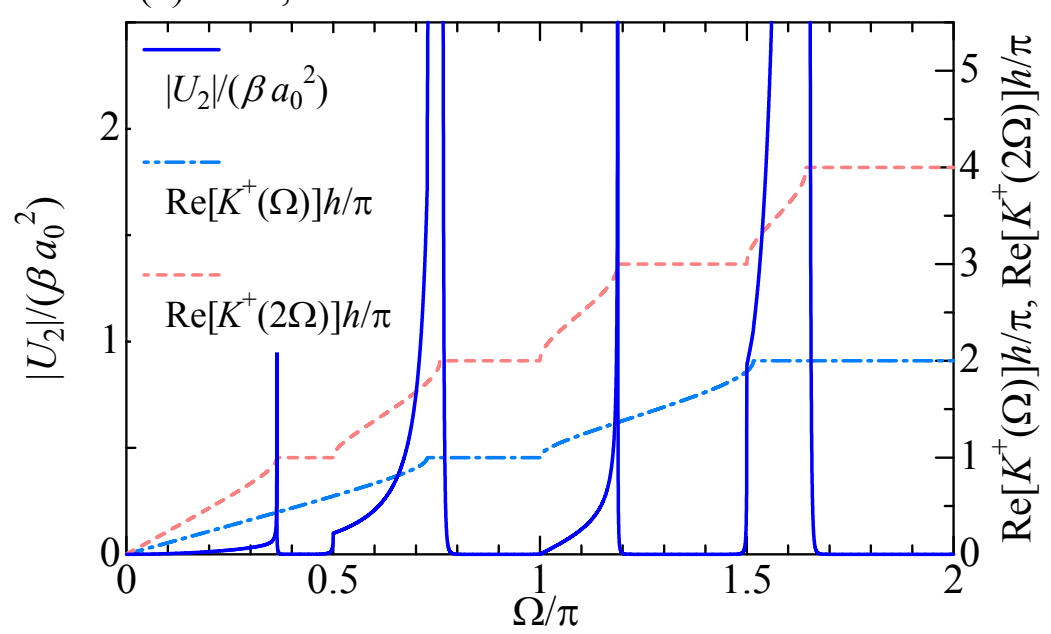

(b) $n=9, m=10$

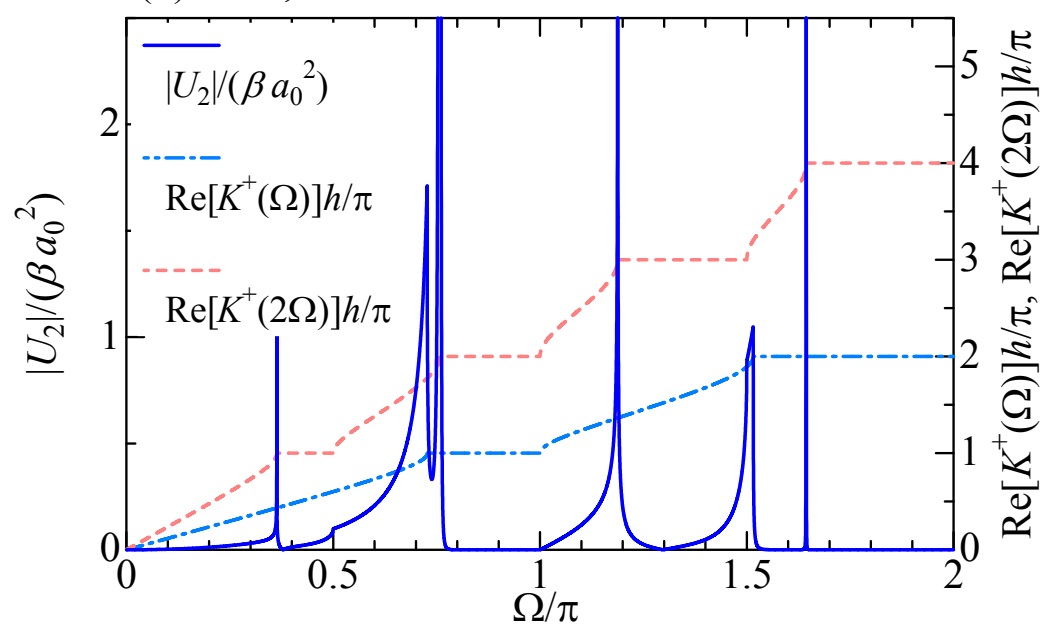

Fig. 4 Frequency dependence of the second-harmonic amplitude at $x=X_{10+}$ when $\Lambda=0.2$ and the nonlinear interface is located at (a) $x=X_{1}$ and (b) $x=X_{9}$. The dispersion curves of the layered structure are also shown. 


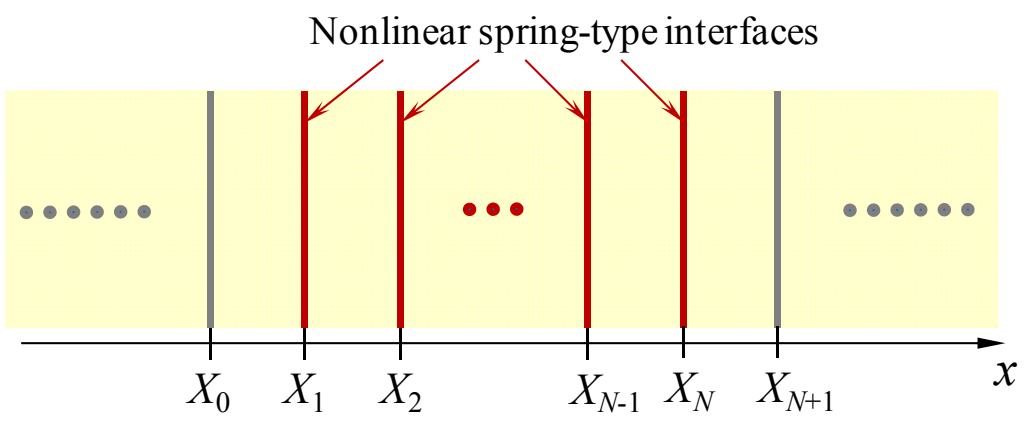

Fig. 5 A schematic for the second-harmonic generation by multiple consecutive nonlinear interfaces in the layered structure.
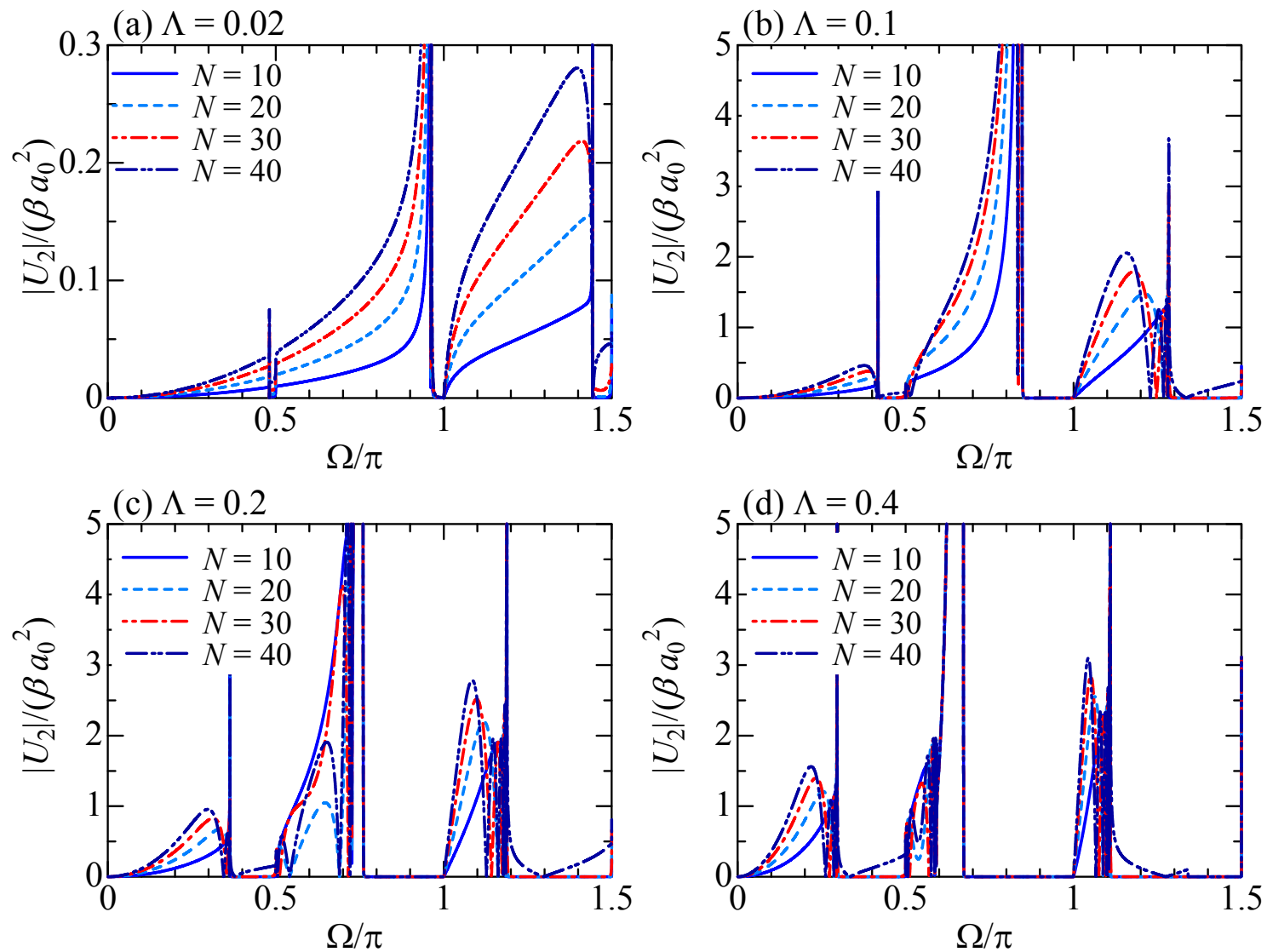

Fig. 6 Frequency dependence of the second-harmonic amplitude at $x=X_{40+}$ for different numbers of nonlinear interfaces $(N=10,20,30,40)$ when (a) $\Lambda=0.02$, (b) $\Lambda=0.1$, (c) $\Lambda=0.2$ and (d) $\Lambda=0.4$. Note that the vertical scale for (a) is different from the others. 

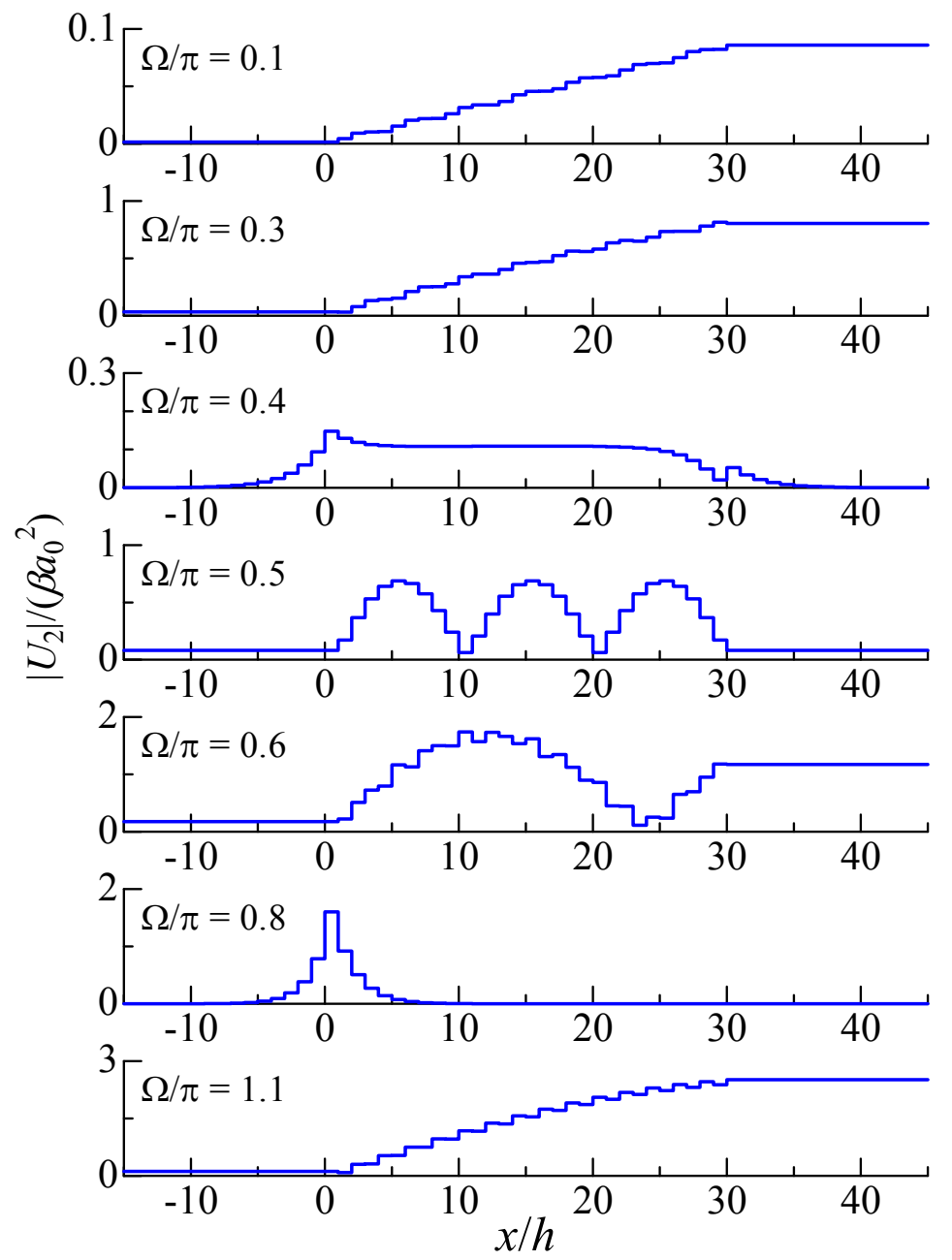

Fig. 7 The second-harmonic amplitude distribution in the layered structure for $\Lambda=0.2$ and different normalized frequencies $\Omega$. Note that the vertical scales are not the same. 

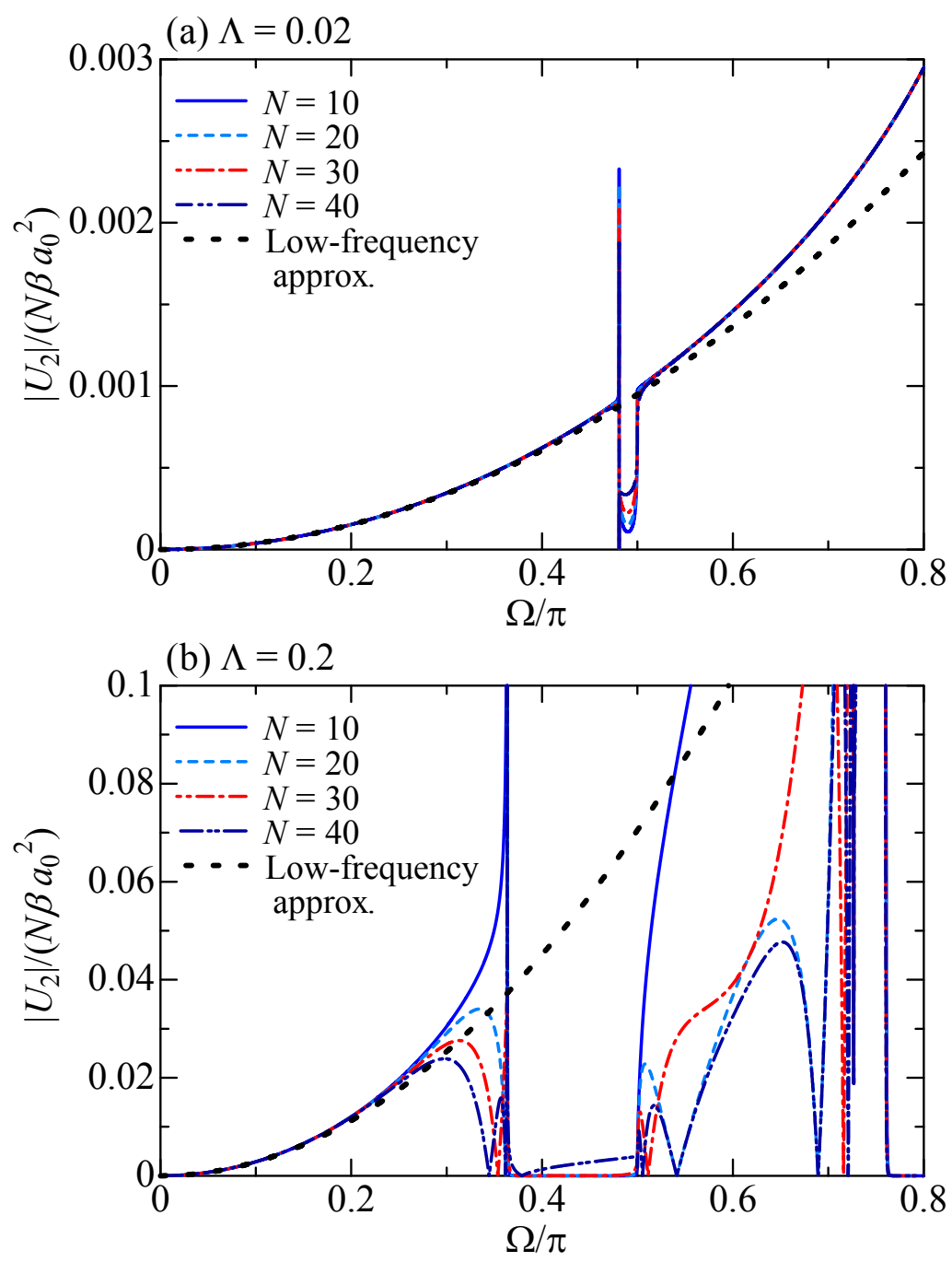

Fig. 8 Frequency dependence of the second-harmonic amplitude at $x=X_{40+}$ for different numbers of nonlinear interfaces $(N=10,20,30,40)$ when (a) $\Lambda=0.02$ and (b) $\Lambda=0.2$, together with the low-frequency approximation. Note that the vertical scales for (a) and (b) are not the same. 